\title{
Three-dimensional analysis of real void closure at the meso-scale during hot metal forming processes
}

\author{
M. Saby ${ }^{\mathrm{a}}$, M. Bernacki ${ }^{\mathrm{a}}$, E. Roux ${ }^{\mathrm{a}}$, P.-O. Bouchard ${ }^{\mathrm{a}}$ \\ ${ }^{a}$ Mines ParisTech, CEMEF - Centre de Mise en Forme des Matériaux, CNRS UMR 7635 CS 102071 rue Claude Daunesse, \\ 06904 Sophia Antipolis cedex, France
}

\begin{abstract}
In the metal forming industry, elimination of internal defects is a prerequisite to avoid catastrophic failure of workpieces. The two different approaches used in the literature to study void closure, respectively at the process-scale and void-scale, present a certain number of limitations. In this paper, an alternative method is presented at a meso-scale. Simulations are performed using a representative volume element (RVE) with real void geometries generated from 3D images obtained using computed microtomography. Complex mechanical loadings are imposed using advanced boundary conditions coming from process numerical modeling. The simulations provide very accurate results to model void closure in real industrial conditions. Equivalent spherical and ellipsoidal voids are also generated and void volume evolution is compared to the case of real geometries. It is shown that the global tendency of void closure is well respected, even though the use of a sphere underestimates void closure, whereas an ellipsoid overestimates void closure, on the final stage. A sensitivity study to mechanical parameters shows that strain-rate has no major influence, while stress triaxiality ratio exhibits a strong influence on the closure of real void. Non-uniform boundary conditions also applied to demonstrate that most existing predictive models are unable to predict void closure in non-uniform mechanical loadings.
\end{abstract}

\section{Introduction}

Industrial needs for large metal components for aerospace, transport, or energy applications constantly increase. During the first steps of elaboration of ingots or preforms, defects, such as voids and internal cavities, may occur. An elimination of these internal defects is required to avoid catastrophic failure during process, or during service of final components. Void elimination is usually performed by means of hot metal forming processes, inducing large deformation in the material at high temperature, and leading to closure of internal voids. Optimization and control of such forming processes in terms of costs and final material soundness remains of prime importance. Void elimination occurs according to two main steps; the mechanical closure, which leads to partial or full contact between internal surfaces, mainly driven by plasticity, and the bonding process, which leads to partial or complete healing at the internal surfaces, mainly driven by diffusion. This paper focuses on the mechanical closure only.

Various studies regarding void closure are described in the literature using approaches at different scales. A first approach, based on analytical micromechanics, considers an isolated void in an infinite medium. Void shape evolution is analytically predicted, in [1] for a linear viscous material, and in [2] for a non-linear viscous material. Spherical and arbitrary ellipsoidal voids are exclusively considered.

Secondly, a macroscopic approach considers a given workpiece containing explicit voids, and is studied according to various working conditions. Qualitative features for processing conditions are presented in [3-6] to foster void closure during hot forging and in [7-10] for hot rolling. Quantitative functions are further

Email address: michel.saby@mines-paristech.fr +33493957415 (M. Saby) 
presented as empirical criteria for void closure applied to hot forging in [11-14] and hot rolling in [11], using finite element simulation and experimental studies. Such finite element simulations typically involve very heavy meshes due to large disparity between the void-scale and the process-scale. Finally, all results from the macroscopic approach remain inevitably process-dependent and it is therefore impossible to precisely understand involved void closure mechanisms, independently from process conditions.

A meso-scale approach is presented in [15] for predicting microstructure-constitutive property relations and is used also in [16] to predict spherical void closure in various loading conditions. In this approach a three-dimensional Representative Volume Element (RVE) is used to perform simulations at the void-scale, using boundary conditions that are representative of macro-scale process conditions.

In this paper, a similar meso-scale approach is used. Now, RVEs are directly generated from real void morphology, obtained using X-ray computed microtomography, or using representative equivalent geometries. Time-dependent loadings are imposed using advanced boundary conditions in order to precisely reproduce industrial conditions. A complete description of the three-dimensional mesh generation and application of boundary conditions is first presented, followed by a convergence study to show validity of the method used. A sensitivity study to mechanical parameters and an analysis of morphology parameters using morphology-equivalent geometries are presented and discussed.

\section{Description of the meso-scale approach}

During hot metal processing, material (and thus internal voids) is submitted to complex mechanical loadings. A detailed knowledge of the local mechanical fields must therefore be assessed. This is performed by simulating the whole industrial process, using the finite element software FORGE ${ }^{\circledR}$. Let us note that the presented approach is illustrated in this paper for a case of hot steel rolling, but can be implemented for any forming process.

It is assumed that the presence of tiny voids has a negligible impact on the global workpiece's deformation at the macroscopic scale. Simulations of hot rolling are thus performed using a void-free workpiece. The values of stress and strain are recorded for given positions using point tracking. The points of interest were determined according to previous statistical observations on industrial cases, and are situated around the workpiece's centerline axis. At the RVE-scale, this assumption means that the RVE dimensions must be small with respect to workpiece dimensions. In other words, the RVE is assimilated as a point in the macroscopic process simulation.

\subsection{Advanced boundary conditions}

It is shown $[11,17]$ that stress triaxiality ratio and equivalent strain have a major influence on void closure. Equivalent strain is expressed as $\varepsilon_{e}=\sqrt{\frac{2}{3} \varepsilon: \varepsilon}$ and stress triaxiality ratio $T_{X}=\frac{\sigma_{m}}{\sigma_{e}}$, where $\sigma_{m}=\frac{1}{3} \operatorname{tr}(\boldsymbol{\sigma})$ is the mean stress and $\sigma_{e}=\sqrt{\frac{3}{2} \boldsymbol{\sigma}: \boldsymbol{\sigma}}$ is the von Mises equivalent stress.

In order to control both strain and stress states in the RVE, boundary conditions are controlled in velocity along $z$-axis and in normal stress along $x$ and $y$-axes. The RVE is a cuboid that comprises three symmetry planes $(O x y),(O y z)$ and $(O z x)$. The boundary conditions are applied on the three other planes of the RVE as presented in Fig 1, where $D_{1}, D_{2}$ and $D_{3}$ are the RVE dimensions in the $x, y$ and $z$ directions, respectively.

Values for $x x$-, $y y$-stress and $z z$-strain, obtained in the RVE are compared to the values measured in the macroscopic hot rolling simulation in Fig. 2. The figure shows that the mechanical conditions from hot rolling are reproduced in the RVE with very good accuracy in the three dimensions. The values for stress triaxiality ratio vs. equivalent strain are also compared in Fig. 2(d). Apart from the slight difference observed for the highest strain values, the figure shows that the complex evolution of stress triaxiality ratio $v s$. equivalent strain is represented in the RVE with very good accuracy. 


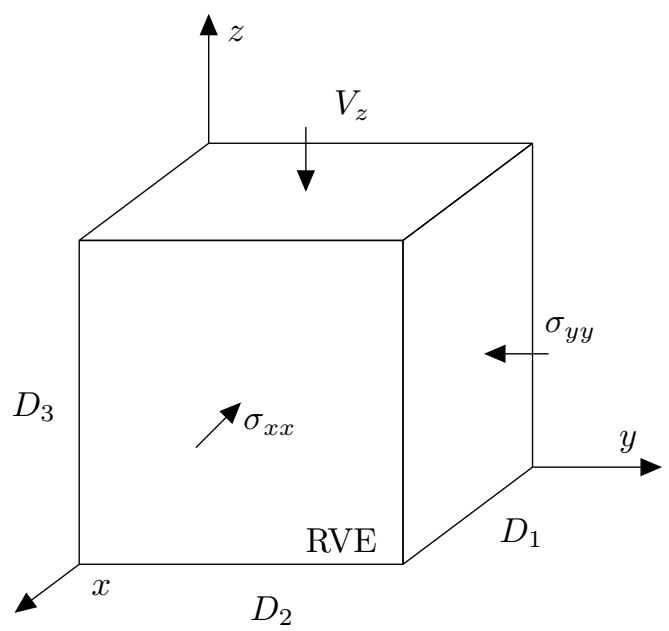

Figure 1: Representative Volume Element, with imposed boundary conditions

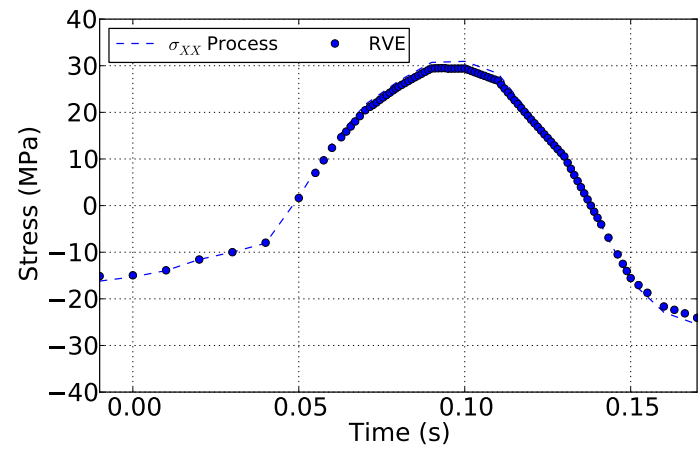

(a) $x x$-stress vs. time

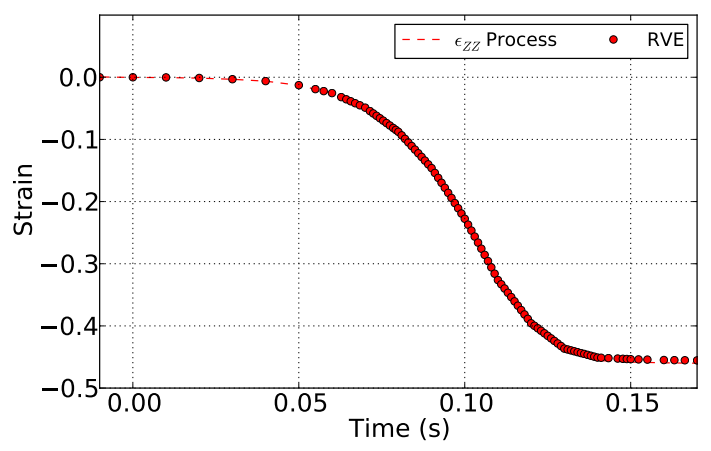

(c) $z z$-strain vs. time

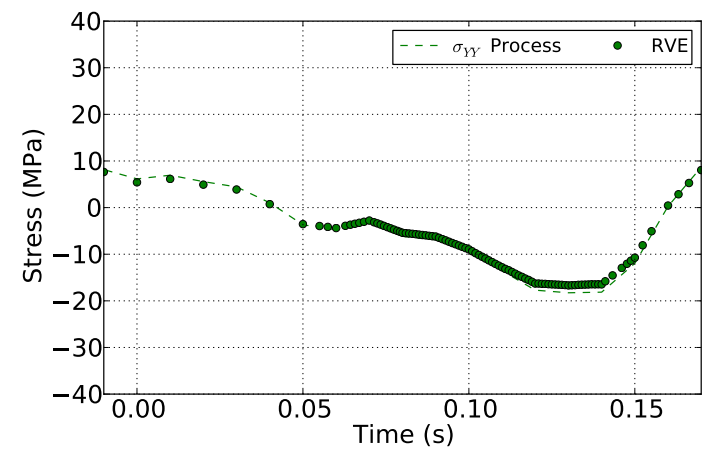

(b) $y y$-stress vs. time

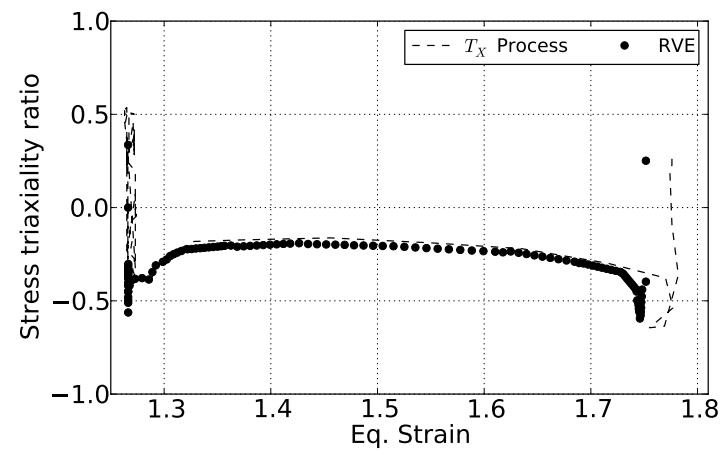

(d) Stress triaxiality ratio vs. equivalent strain

Figure 2: Comparison of mechanical variables in the RVE with values from point tracking in hot rolling process 


\subsection{Generation of a 3D-mesh containing real void geometry}

Porosity was identified in a typical industrial steel ingot. A cylindrical specimen containing several voids was extracted (diameter $20 \mathrm{~mm}$ ) for non-destructive examination. A three-dimensional image of the sample was obtained using X-ray computed microtomography using a Y.TU 450-D09 X-ray tube with acceleration tension of $220 \mathrm{kV}$. The detector type was Y.XRD820, focal-detector distance $1123.0 \mathrm{~mm}$ and focal-specimen distance $723.2 \mathrm{~mm}$, leading to magnification of 1.55 . The number of recorded projections was 810 and 3D-reconstruction provided an isotropic voxel size of $0.128 \mathrm{~mm}$.

Raw data were then submitted to a 3D median filter, in order to reduce the grayscale noise and further artifacts. A three-dimensional surface mesh of the matrix-void interface was then generated, by means of an algorithm based on the Marching Cubes method [18, 19]. According to this algorithm, the position of mesh nodes is directly determined by the voxel spatial periodicity. Therefore, the obtained surface mesh has a homogeneous mesh size, which corresponds to the isotropic voxel size of the initial image $(0.128 \mathrm{~mm})$.

From the obtained surface mesh, a 3D volume conforming mesh is generated in a cuboid and the finite element mesh of the RVE containing real void morphology is obtained, see Fig. 3. The RVE dimension in each spatial direction is determined with a proportionality coefficient $n$ from the dimensions of the void.

$$
D_{i}=n \times d_{i} \quad, \quad i \in[1,3],
$$

where $d_{i}$ are the void dimensions in the $i$ direction.

A viscoplastic constitutive model with strain-hardening, typical for hot steels, is used in isothermal conditions. Self-contact at the void's interface during deformation is controlled by a master-slave algorithm for deformable bodies. It is based on the penalty method, involving a no-friction and unilateral contact, as described in [20].

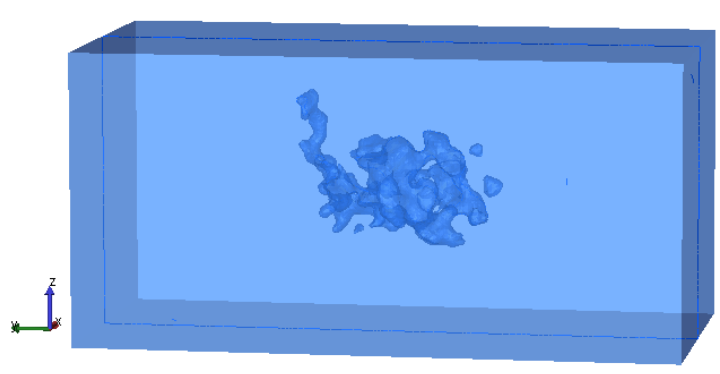

(a) Initial state

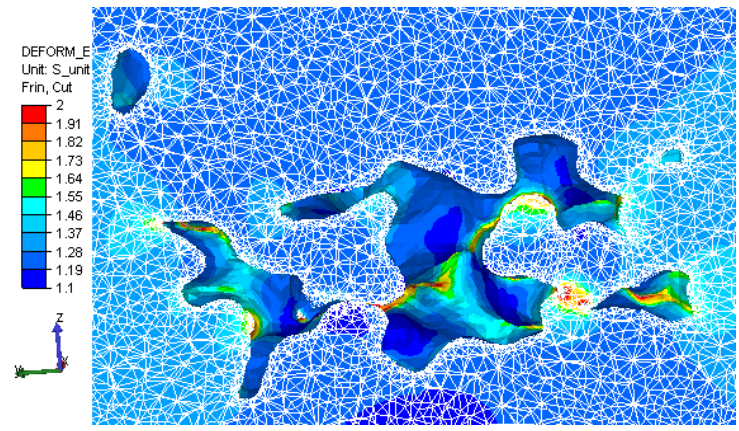

(b) Cutting plane at $14 \%$ height reduction

Figure 3: RVE with dimensions given in Eq. 1 with $n=3$, containing VOID1

\subsection{Convergence study of RVE simulations}

\subsubsection{Mesh size}

The mesh topology at the internal void surface remains fixed due to initial mesh conformity. A coarse mesh size $\left(h_{\max }=1 \mathrm{~mm}\right)$ is set in the bulk and a finer mesh size $h_{\min }$ is defined around the void. A sensitivity study to $h_{\min }$ was performed and exhibited a relatively strong dependence on mesh size. The determination of an optimal mesh size will enable ensuring convergence of the simulations results, as well as reducing CPU computation time. RVE dimensions are fixed using the value $n=3$ in Eq. 1. Various test values were used and are given in Table 1. For each mesh size, the void volume evolution in time is plotted in Fig. 4(a). This figure highlights the dependence in mesh size, leading to a maximum difference value of about $10 \%$ on the final void volume between the coarsest and the finest values. This difference may be explained by strong local fluctuations of the mechanical fields around the void that require sufficient mesh refinement, see Fig. 3(b). Fig. 4(b) shows that the values converge for $h_{\min } \leqslant 0.1 \mathrm{~mm}$. The relative 
difference obtained between results at $0.1 \mathrm{~mm}$ and those at $0.05 \mathrm{~mm}$ is lower than $0.5 \%$, while the CPU time is multiplied by a factor greater than 3 . The value $h_{\min }=0.1 \mathrm{~mm}$ is considered as an acceptable value and will be used in all simulations presented in this paper.

\begin{tabular}{|c|c|c|c|}
\hline$h_{\min }(\mathrm{mm})$ & Nb nodes & Nb CPUs & CPU time \\
\hline \hline 1 & 12000 & 2 & 30 min \\
\hline 0.7 & 14000 & 1 & 2 hours \\
\hline 0.5 & 19000 & 2 & 2 hours \\
\hline 0.4 & 23000 & 2 & 6 hours \\
\hline 0.3 & 32000 & 2 & 10 hours \\
\hline 0.2 & 67000 & 4 & 12 hours \\
\hline 0.1 & 400000 & 16 & 50 hours \\
\hline 0.05 & 650000 & 16 & 165 hours \\
\hline
\end{tabular}

Table 1: Tested mesh sizes with associated computation features

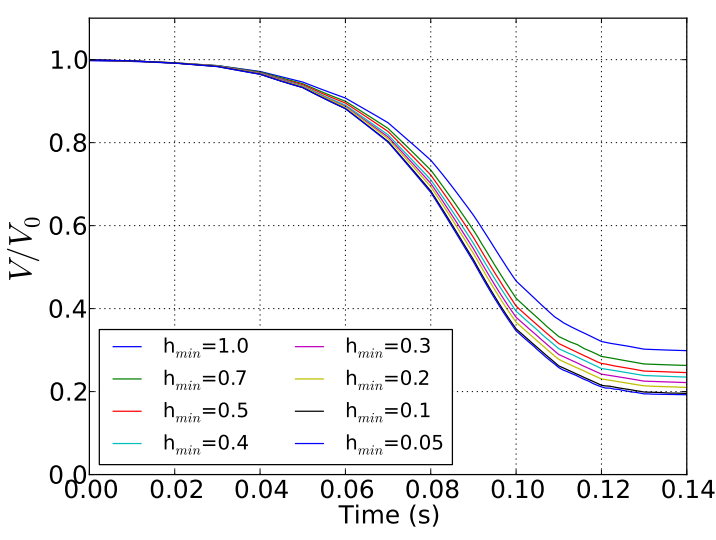

(a) Void volume evolution

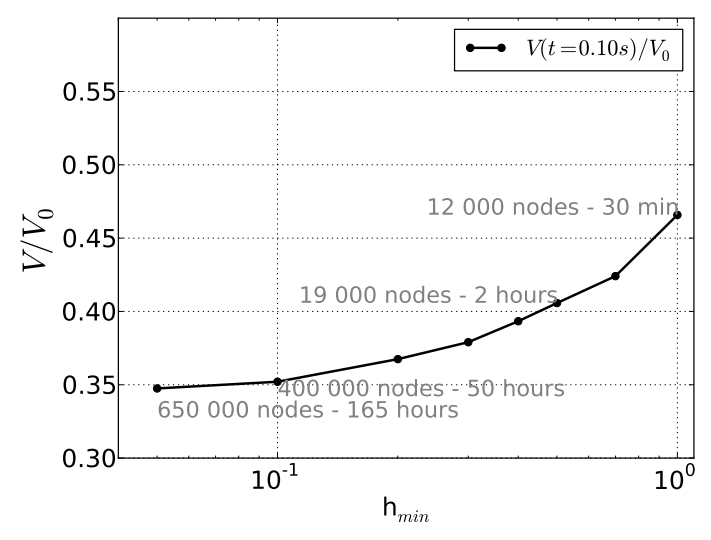

(b) Void volume at $t=0.10 \mathrm{~s} v s$. mesh size

Figure 4: Effect of mesh size on VOID1 volume evolution in hot rolling conditions

\subsubsection{RVE Dimensions}

Dimensions of the RVE are minimized in order to better satisfy the assumption made by the multiscale approach, and to reduce CPU time. Several RVEs were generated using different values of $n$ and the influence of $n$ on void volume evolution was observed. Fig. 5(a) shows that the volume evolutions obtained with $n=2$ underestimates void closure of about $8 \%$ compared to values obtained with $n=5$. This difference is mainly due to boundary effects that arise when boundaries are too close from the void. For the case $n=3$, the difference in void volume is lower than $1.5 \%$ with respect to the case $n=5$, which is acceptable. The simulations presented in this paper are performed with $n=3$.

\subsection{Verification of the void volume prediction in the $R V E$}

A full explicit simulation of the hot rolling case containing VOID1 was performed in order to compare the results with the ones obtained at the RVE scale. The mesh size around the void in the billet is set at the value $h_{\min }=0.1 \mathrm{~mm}$ in order to be consistent with the results regarding mesh size in the RVEs. The mesh size is gradually coarser in the rest of the billet in order to reduce the total number of elements $\left(h_{\max }=30 \mathrm{~mm}\right)$. The computation time was about 11 days on 32 CPUs. The void volume evolution obtained in the explicit process simulation is plotted in Fig. 5(b), compared to the one obtained in the RVE. Let us note that remeshing difficulties were encountered during the explicit process computation due to the large disparity of mesh size between the zone containing the void and the rest of the billet. The computation 


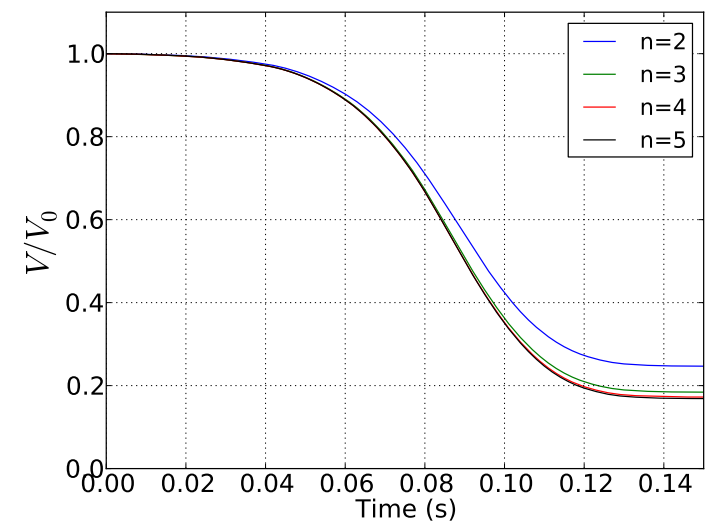

(a) Void volume evolution for various RVE dimensions (b) Comparison of void volume evolution in the explicit process simulation and in the RVE $(n=3)$

Figure 5: Effect of RVE dimensions on VOID1 volume evolution in hot rolling conditions

stopped around $t=0.11 \mathrm{~s}$ due to mesh degeneration. However, the two evolutions show very good agreement on the whole computed range. This result shows that the use of an RVE with boundary conditions is able to represent the behavior of voids in real conditions with very good accuracy. In terms of computation time, the simulation at the RVE scale was about 10 times faster than the explicit hot rolling simulation.

\section{Sensitivity to mechanical parameters}

The RVE containing VOID1 was used to perform a sensitivity study to two mechanical parameters: strain-rate and stress triaxiality ratio. A constant strain-rate is applied along the $z$-axis by imposing velocity on the upper face of the RVE. Stress values along $x$ and $y$-axes are controlled in order to provide a constant stress triaxiality ratio during deformation. In Fig. 6, void volume evolution is plotted for different values of stress triaxiality ratio $(-0.15$ to -0.82$)$, and for various values of strain-rate $(0.001 / \mathrm{s}$ to $100 / \mathrm{s})$. The figure shows a strong influence of stress triaxiality ratio on void closure. From the earliest stage of deformation, closure is more effective for lower triaxiality ratios. This result verifies the main results obtained in the literature using spherical voids [1].

Due to the viscoplastic behavior of the material, the flow stress varies from about $24 \mathrm{MPa}$ to $140 \mathrm{MPa}$ on the range of tested strain-rate. The resulting fluctuations in flow stress due to the fluctuations of strain values around the void might thus influence the material's flow around the void and have an impact on void closure. In fact, this impact is not significant and the figure shows that the difference due to strain-rate is negligible compared to the difference due to stress triaxiality ratio.

\section{Analysis of morphology parameters}

In most studies regarding void closure in the literature, spherical and arbitrary ellipsoidal voids are usually considered in a first approximation $[1,2,6,16,17]$. However, industrial observations usually exhibit rather complex morphologies, such as VOID1. An evaluation of the error made by using equivalent geometries instead of real void is thus presented in this section.

In this work, ellipsoids are generated in order to precisely respect shape factors and principal orientations of real voids. Morphology parameters are computed using the inertia matrix (Eq. 2) of the binarized 3Dimage of an initial void.

$$
\left(\begin{array}{ccc}
I_{o x} & -I_{x y} & -I_{x z} \\
-I_{x y} & I_{o y} & -I_{y z} \\
-I_{x z} & -I_{y z} & I_{o z}
\end{array}\right)
$$




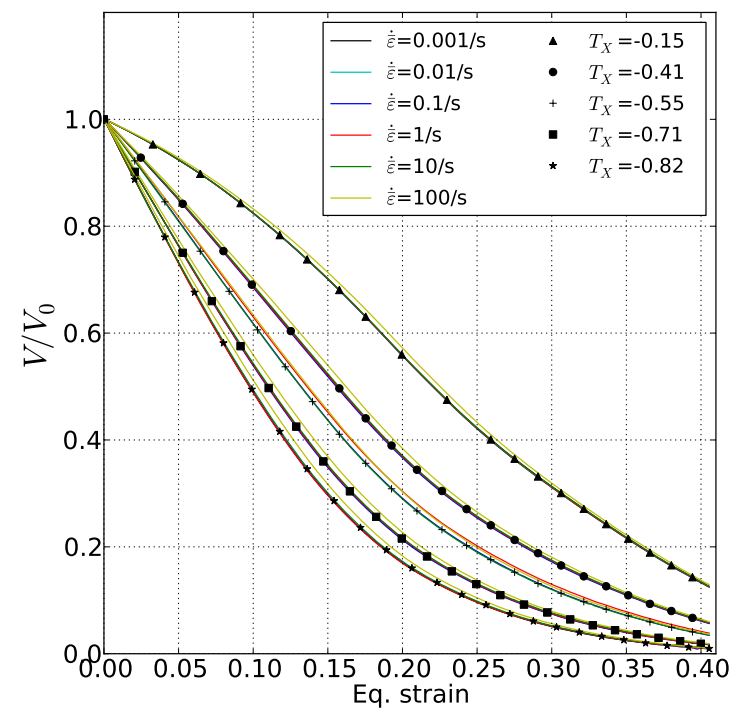

Figure 6: Void volume evolution in various mechanical conditions

$$
\begin{aligned}
I_{o x} & =\sum m_{i}\left(y_{i}^{2}+z_{i}^{2}\right) \\
\text { with } I_{o y} & =\sum m_{i}\left(x_{i}^{2}+z_{i}^{2}\right) \\
I_{o z} & =\sum m_{i}\left(x_{i}^{2}+y_{i}^{2}\right)
\end{aligned} \text { and } \begin{aligned}
I_{x y} & =\sum m_{i}\left(x_{i} \cdot y_{i}\right) \\
& =\sum m_{i}\left(y_{i} \cdot z_{i}\right) . \\
I_{x z} & =\sum m_{i}\left(x_{i} \cdot z_{i}\right)
\end{aligned} .
$$

The sum is performed on the voxels of the 3D-image, with $x_{i}, y_{i}$ and $z_{i}$ the coordinates of the $i^{\text {th }}$ voxel, and $m_{i}$ its binary value $\left(m_{i}=1\right.$ if voxel is inside void, $m_{i}=0$ elsewhere).

Diagonalization of the inertia matrix provides eigen-values and eigen-vectors that are used to generate an equivalent ellipsoid. Initial void volume is also respected using a normalization factor.

A sphere was generated as well. Its diameter is defined such that its volume equals the initial volume of the real void.

The morphology-equivalent ellipsoid is shown in Fig. 7(a) with green color, superimposed with the real void in blue color and the volume-equivalent sphere in red color. The imposed boundary conditions are the ones described in paragraph 2.1 for hot rolling.

Fig. 7(b) presents the void volume evolutions for the three initial geometries. The three curves exhibit a common tendency, reaching maximal closure around $t=0.13 \mathrm{~s}$. The behavior of each curve is mainly driven by the boundary conditions that are rather complex in this case. Discrepancies may however be pointed out between the three curves and are discussed. At the first stage of deformation, void closure is slightly underestimated for both equivalent geometries. This effect may be attributed to the elevated tortuosity of the real morphology, which is not taken into account in the equivalent geometries. The tortuosity comprises a certain quantity of cavities that present various initial shapes. Some of them must be favorable to void closure.

During the total deformation this difference (about 8-12\%) remains rather constant in the case of the sphere, while the tendency is reversed at $t>11 \mathrm{~s}$ in the ellipsoidal case. The final volume overestimates the real void of about $7 \%$. That difference may be attributed to morphology parameters that are not taken into account in the spherical case. For further analysis, a separation of the morphology effects and influence of mechanical loadings is required. Additionnally, VOID1 presents a rather equiaxed morphology, inducing a relative small difference between spherical and ellipsoidal predictions.

A second RVE containing VOID2 was then generated using the same methodology. In this case, constant boundary conditions are applied on the RVE: $\varepsilon_{z z}=0.1 / s$ and $\sigma_{x x}=\sigma_{y y}=20 \mathrm{MPa}$. This way, the study exclusively focuses on morphological aspects of VOID2. A morphology-equivalent ellipsoid and a volumeequivalent sphere were also generated for VOID2, see Fig. 8(a). The volume evolution of each case is plotted 
in Fig. 8(b).

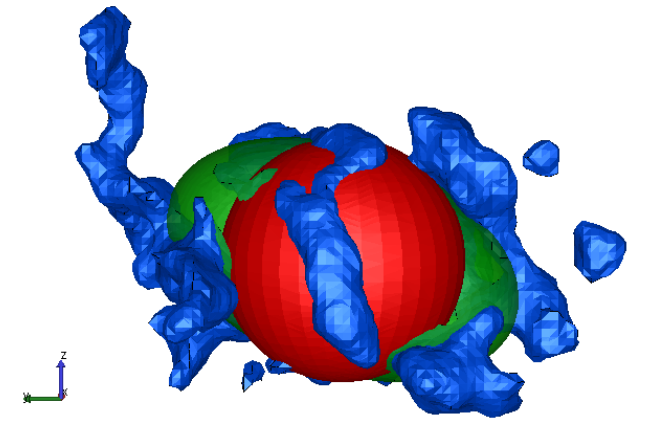

(a) Initial void interfaces for VOID1 and equivalent geometries

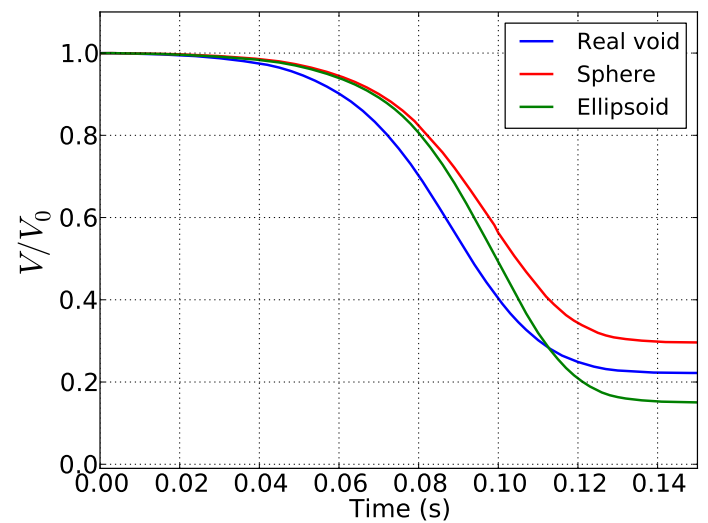

(b) Volume of VOID1 in real hot rolling conditions

Figure 7: Equivalent geometries for VOID1

Again, the spherical void underestimates real closure during the whole deformation, with a relative difference up to about 30\% compared to real void. However, the ellipsoidal void shows a good agreement with the real void. The relative difference remains lower than $2 \%$ on the range $t=[0-1.5 s]$, i.e. over about $70 \%$ of void closure phenomenon. Similarly to results on VOID1, the equivalent ellipsoid overestimates void closure after a certain time (here $t=1.5 \mathrm{~s}$ ), up to a maximum difference of about $-11 \%$ at $t=2.5 \mathrm{~s}$. The use of an equivalent ellipsoid thus provides better prediction than the use of a volume-equivalent sphere. Let us finally note that complete closure of real void was not reached at the end of the simulation $(t=6 s)$, while both equivalent geometries are completely closed up at this time. This underlines the impact of tortuosity at the final stage of closure. Tiny residual voids remain difficult to be closed up, even at high deformation rates.

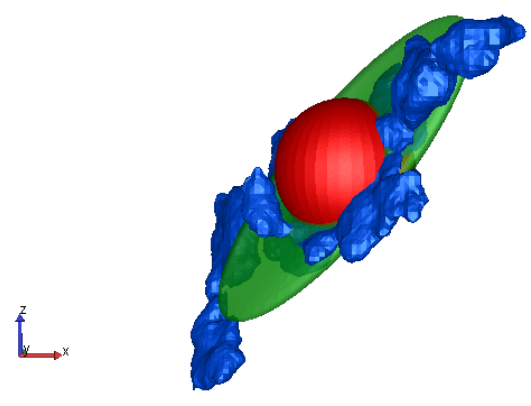

(a) Initial void interface for VOID2 and equivalent geometries

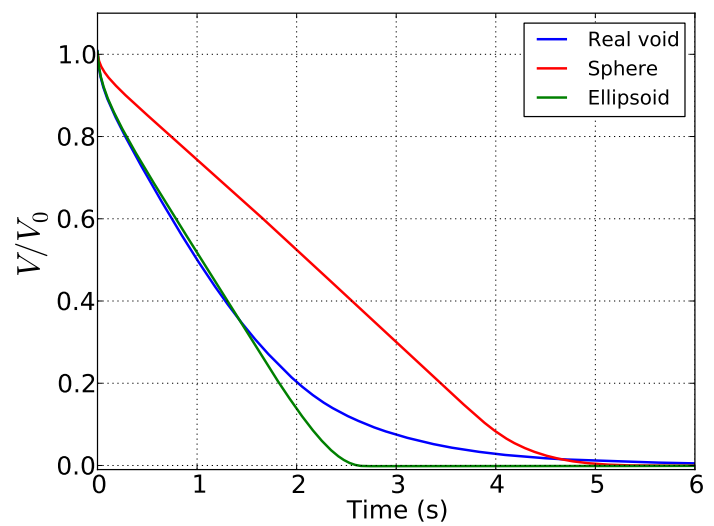

(b) Volume of VOID2 in constant boundary conditions

Figure 8: Equivalent geometries for VOID2

It is worth noticing that VOID1 presents a rather equiaxed morphology, with a principal orientation that is roughly perpendicular to the deformation axis (z-axis), whereas VOID2 presents a rather flat initial 
shape, with a principal orientation making an angle of about $45^{\circ}$ with the deformation axis. In the case of non-equiaxed voids, the use of an equivalent ellipsoid provides a better prediction than a volume-equivalent sphere.

\section{Effect of non-uniform loadings}

In this section, time dependent boundary conditions are applied in order to induce non-uniform loadings in the material. The RVE containing VOID2 is used and velocity is successively imposed along $z$ - and $x$-axes, as presented in Tab. 2. Case A corresponds to a constant compression along $z$-axis. In the case $\mathrm{B}$, compression along $z$-axis is interrupted at $t=1 s$ and is switched to $x$-axis. In the case $\mathrm{C}$, compression direction is successively switched from $z$ - to $x$ - and back to $z$-axis. Cases D, E and F are similar to case B, but switching compression direction later at $2 s, 3 s$ and $5 s$ respectively. During deformation, the value of imposed velocity is adjusted in order to apply a constant macroscopic strain-rate $\left(\dot{\bar{\varepsilon}}=0.1 s^{-1}\right)$ throughout the deformation duration. Stress boundary conditions on the perpendicular faces to compression direction are set to zero, involving a constant stress triaxiality ratio $T_{X}=-1 / 3$ throughout deformation duration.

Four different RVEs were used (see Fig. 9): (a) the RVE containing real void morphology VOID2, (b) the RVE containing the morphology-equivalent ellipsoid, (c) the RVE containing the volume-equivalent sphere, and (d) an RVE free of void, in which a simple closure prediction model is implemented, expressed by:

$$
\frac{d V}{d t}=V_{0} k T_{X} \dot{\bar{\varepsilon}}
$$

where $k$ is a constant parameter of the model and $V_{0}$ the initial void volume. This simple model is consistent with the ones from literature $[1,2,11-14,16]$ in the sense that a volume variation is expressed as a function of scalar variables (equivalent strain and stress triaxiality ratio).

\begin{tabular}{|c|c|c|c|c|c|}
\hline Interval & {$[0-1 s]$} & {$[1-2 s]$} & {$[2-3 s]$} & {$[3-5 s]$} & {$[5-10 s]$} \\
\hline \hline Case A & $Z$ & $Z$ & $Z$ & $Z$ & $Z$ \\
\hline Case B & $Z$ & $X$ & $X$ & $X$ & $X$ \\
\hline Case C & $Z$ & $X$ & $Z$ & $Z$ & $Z$ \\
\hline Case D & $Z$ & $Z$ & $X$ & $X$ & $X$ \\
\hline Case E & $Z$ & $Z$ & $Z$ & $X$ & $X$ \\
\hline Case F & $Z$ & $Z$ & $Z$ & $Z$ & $X$ \\
\hline
\end{tabular}

Table 2: Compression axes for all tested cases for non-uniform loadings

The results show that switching compression direction has a significant impact on void closure ratio. The strongest effect is shown in Fig. 9(c) for the spherical case with a significant change of slope at each switching direction. The $z$-axis compression deforms the initially spherical shape into an oblate shape, which therefore becomes harder to close in a perpendicular direction. Case F illustrates this effect at an extreme point. At $t=5 \mathrm{~s}$, the void is transformed into a penny-like shape that is particularly unfavorable to be closed up along $x$-axis compression. This leads to a slightly positive slope of the void volume evolution.

In the case of real void compression (see Fig. 9(a)), the change of slope at any switching instant is much smaller than in the spherical case. This is due to the fact that the real void morphology initially exhibits a strong anisotropy and that its principal orientation does never coincide with any compression direction. A direct link can therefore be pointed out between the compression direction and the void's principal orientation. In the case of equivalent ellipsoid, morphological parameters are taken into account, and the resulting changes of slope in Fig. 9(b) is comparable to the ones in Fig. 9(a). This demonstrates the interest of using equivalent ellipsoid to substitute a real void morphology instead of a sphere.

The void volume prediction in Fig. 9(d) shows a linear evolution of void volume. This naturally comes from the linear combination of mechanical variables given in Eq. 3, which are intentionally kept constant during deformation. More interestingly, the model is not able to predict any change in void volume evolution during switching compressions. This is implicitly due to its definition that is based on equivalent variables $T_{X}$ and $\dot{\bar{\varepsilon}}$. In fact, all existing prediction models cited in the introduction of this paper are based on these 
two mechanical equivalent variables as well. None of them shall therefore be able to predict changes in void closure according to non-uniform loadings.

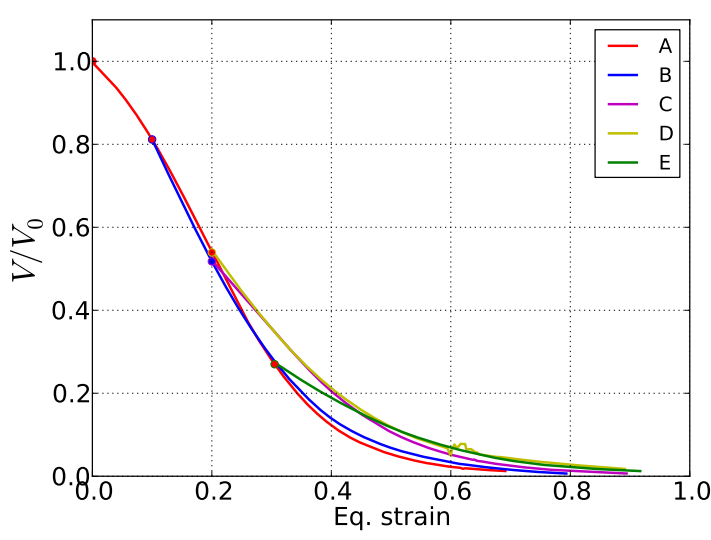

(a) RVE containing real morphology of VOID2

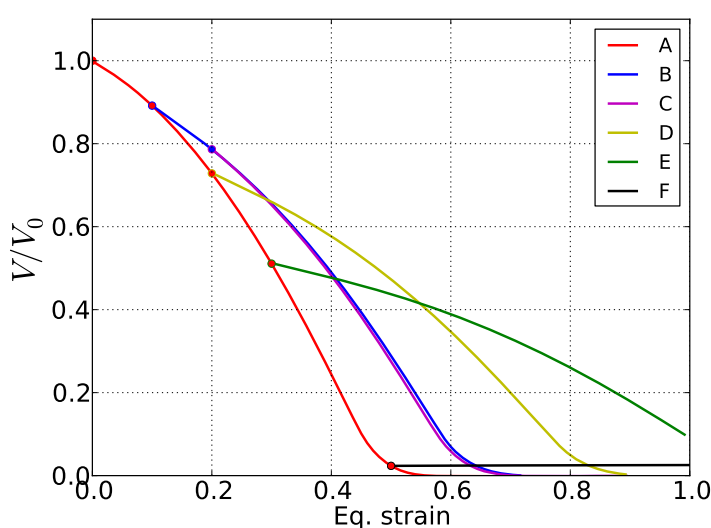

(c) RVE containing volume-equivalent sphere

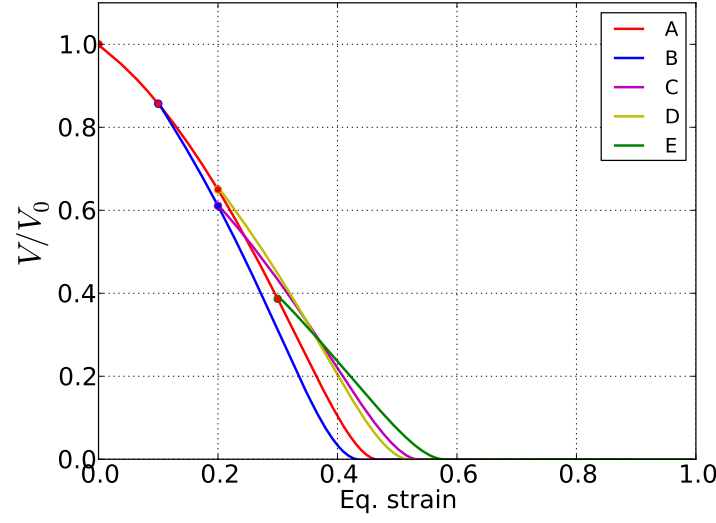

(b) RVE containing morphology-equivalent ellipsoid

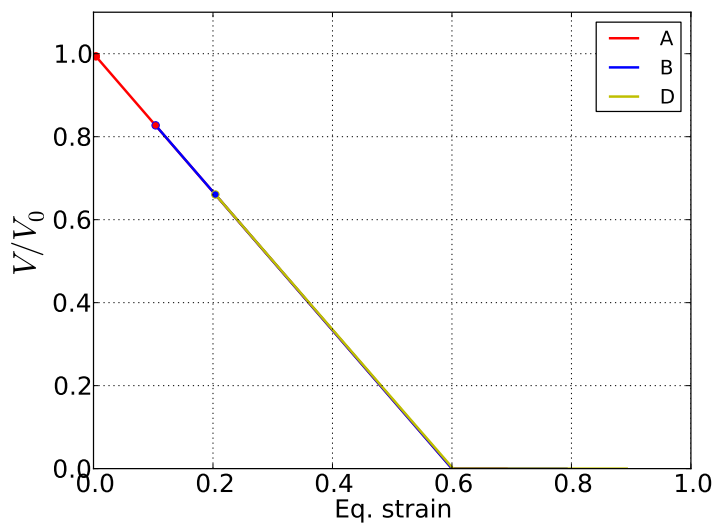

(d) Void-free RVE with prediction model from Eq. 3

Figure 9: Non-uniform compression cases

\section{Conclusions}

A meso-scale approach was successfully implemented to simulate closure of real voids in complex mechanical conditions. Industrially-representative loadings are reproduced in a representative volume element using advanced boundary conditions and show very good agreement. X-ray computed microtomography was used to obtain real void morphologies and a mesh of a cubic RVE containing a real void was generated with very good accuracy. Mesh refinements and dimensions of the RVE were determined to enable convergence of simulations' results.

A sensitivity study to mechanical parameters has shown that stress triaxiality ratio has a strong influence on the closure of a real void. This result shows good agreement with the results obtained in the literature using simple geometries. However, strain-rate was shown to have a negligible influence on void closure, despite the strong visco-plastic behavior of the material.

The generation of a void's morphology-equivalent ellipsoid, respecting volume, shape factors and principal orientations, was successfully implemented. A comparison between real morphology, sphere and ellipsoid provides a good estimation of the error made by the use of equivalent geometries. The use of a sphere to 
predict void closure was rather limited in the case of a void presenting a non-equiaxed morphology, while the use of an equivalent ellipsoid shows better prediction. This effect is less marked when the void tends to show an equiaxed morphology.

The initial tortuosity mostly shows an effect on the final stage of closure.

A significant influence of morphology parameters on void closure was shown in the case of non-uniform compression. The interest of considering changes in void shape during deformation in order to accurately predict its volume evolution was demonstrated. Current prediction models involving equivalent mechanical variables are not able to predict changes in volume evolution due to non-uniform deformation.

In future work, the influence of morphological parameters must be qualitatively studied. Cross-influences with stress triaxiality and principal deformation axes may be also pointed out in order to propose a new model. That model may take into account the influence of initial void morphology on the void volume evolution. A prediction of the morphology evolution during deformation is the next step to be able to predict changes due to non-uniform compressions.

\section{Acknowledgments}

The authors would like to gratefully thank Timet Savoie, as well as Aubert\&Duval, Ascometal, Creusot Forge (Areva), Industeel (Arcelormittal) and Constellium for funding this research, and Cetim (Pôle EPI) for micro-tomography observations.

\section{References}

[1] B. Budiansky, J. W. Hutchinson, and S. Slutsky, Void growth and collapse in viscous solids, pp. 13-45. Pergamon Press, Oxford, 1982.

[2] B. Lee and M. Mear, "Studies of the growth and collapse of voids in viscous solids," Journal of Engineering Materials and Technology, Transactions of the ASME, vol. 116, no. 3, pp. 348-358, 1994.

[3] S. Dudra and Y.-T. Im, "Analysis of void closure in open-die forging," International Journal of Machine Tools and Manufacture, vol. 30, no. 1, pp. 65-75, 1990.

[4] G. Banaszek and A. Stefanik, "Theoretical and laboratory modelling of the closure of metallurgical defects during forming of a forging," Journal of Materials Processing Technology, vol. 177, no. 1-3, pp. 238-242, 2006.

[5] M. S. Chun, C. J. Van Tyne, and Y. H. Moon, "FEM analysis of void closure behaviour during open die forging of rectangular billets," Steel Research International, vol. 77, no. 2, pp. 116-121, 2006

[6] Y. D. Kim, J. R. Cho, and W. B. Bae, "Efficient forging process to improve the closing effect of the inner void on an ultra-large ingot," Journal of Materials Processing Technology, vol. 211, no. 6, pp. 1005-1013, 2011.

[7] A. Wallerö, "Closing of a central longitudinal pore in hot rolling," Journal of Mechanical Working Technology, vol. 12, no. 2, pp. 233-242, 1985.

[8] M. Pietrzyk, R. Kawalla, and H. Pircher, "Simulation of the behaviour of voids in steel plates during hot rolling," Steel Research, vol. 66, no. 12, pp. 526-529, 1995.

[9] Y. M. Hwang and D. C. Chen, "Finite element simulations on void closure behaviour inside the sheet during sheet rolling processes," Proceedings of the Institution of Mechanical Engineers, Part B: Journal of Engineering Manufacture, vol. 216, no. 9, pp. 1227-1237, 2002.

[10] D. C. Chen, "Rigid-plastic finite element analysis of plastic deformation of porous metal sheets containing internal void defects," Journal of Materials Processing Technology, vol. 180, no. 1-3, pp. 193-200, 2006.

[11] M. Nakasaki, I. Takasu, and H. Utsunomiya, "Application of hydrostatic integration parameter for free-forging and rolling," Journal of Materials Processing Technology, vol. 177, no. 1-3, pp. 521-524, 2006.

[12] H. Kakimoto, T. Arikawa, Y. Takahashi, T. Tanaka, and Y. Imaida, "Development of forging process design to close internal voids," Journal of Materials Processing Technology, vol. 210, no. 3, pp. 415-422, 2010.

[13] Y. Lee, S. Lee, C. Van Tyne, B. Joo, and Y. Moon, "Internal void closure during the forging of large cast ingots using a simulation approach," Journal of Materials Processing Technology, vol. 211, no. 6, pp. 1136-1145, 2011.

[14] K. Chen, Y. Yang, G. Shao, and K. Liu, "Strain function analysis method for void closure in the forging process of the large-sized steel ingot," Computational Materials Science, vol. 51, no. 1, pp. 72-77, 2012.

[15] X. Yin, W. Chen, A. To, C. McVeigh, and W. K. Liu, "Statistical volume element method for predicting microstructureconstitutive property relations," Computer Methods in Applied Mechanics and Engineering, vol. 197, no. 43-44, pp. 3516$3529,2008$.

[16] X.-X. Zhang, Z.-S. Cui, W. Chen, and Y. Li, "A criterion for void closure in large ingots during hot forging," Journal of Materials Processing Technology, vol. 209, no. 4, pp. 1950-1959, 2009.

[17] J. Kim, X. Gao, and T. S. Srivatsan, "Modeling of void growth in ductile solids: effects of stress triaxiality and initial porosity," Engineering Fracture Mechanics, vol. 71, no. 3, pp. 379-400, 2004. 
[18] W. E. Lorensen and H. E. Cline, "Marching cubes: A high resolution 3d surface construction algorithm," SIGGRAPH Comput. Graph., vol. 21, pp. 163-169, 1987.

[19] D. Rajon and W. Bolch, "Marching cube algorithm: Review and trilinear interpolation adaptation for image-based dosimetric models," Computerized Medical Imaging and Graphics, vol. 27, no. 5, pp. 411-435, 2003.

[20] L. Fourment, J. Barboza, and S. Popa, "Master/slave algorithm for contact between deformable bodies and axial symmetries - application to 3D metal forging," in Computational Fluid and Solid Mechanics 2003 (K. Bathe, ed.), pp. 269-272, Oxford: Elsevier Science Ltd, 2003. 